\title{
Structural Properties of One-Way Hash Functions
}

\author{
Yuliang Zheng \\ Tsutomu Matsumoto \\ Hideki Imai
}

\author{
Division of Electrical and Computer Engineering \\ Yokohama National University \\ 156 Tokiwadai, Hodogaya, Yokohama, 240 JAPAN
}

\begin{abstract}
We study the following two kinds of one-way hash functions: universal oneway hash functions (UOHs) and collision intractable hash functions (CIHs). The main property of the former is that given an initial-string $x$, it is computationally difficult to find a different string $y$ that collides with $x$. And the main property of the latter is that it is computationally difficult to find a pair $x \neq y$ of strings such that $x$ collides with $y$. Our main results are as follows. First we prove that $\mathrm{UOHs}$ with respect to initial-strings chosen arbitrarily exist if and only if UOHs with respect to initial-strings chosen uniformly at random exist. Then, as an application of the result, we show that UOHs with respect to initial-strings chosen arbitrarily can be constructed under a weaker assumption, the existence of one-way quasi-injections. Finally, we investigate relationships among various versions of one-way hash functions. We prove that some versions of one-way hash functions are strictly included in others by explicitly constructing hash functions that are one-way in the sense of the former but not in the sense of the latter.
\end{abstract}

\section{Introduction}

One-way hash functions are a principal primitive in cryptography. There are roughly two kinds of one-way hash functions: universal one-way hash functions (UOHs) and collision intractable hash functions (CIHs). The main property of the former is that given an initial-string $x$, it is computationally difficult to find a different string $y$ A.J. Menezes and S.A. Vanstone (Eds.): Advances in Cryptology - CRYPTO '90, LNCS 537, pp. 285-302, 1991. (C) Springer-Verlag Berlin Heidelberg 1991 
that collides with $x$. And the main property of the latter is that it is computationally difficult to find a pair $x \neq y$ of strings such that $x$ collides with $y$. Naor and Yung constructed UOHs under the assumption of the existence of one-way injections (i.e., one-way one-to-one functions) [NY89], and Damgård constructed CIHs under a stronger assumption, the existence of claw-free pairs of permutations [Dam89]. In [NY89], Naor and Yung also presented a general method for transforming any UOH into a secure digital signature scheme. We are interested both in constructing UOHs under weaker assumptions and in relationships among various versions of one-way hash functions. Our main results are summarized as follows.

First, we prove that UOHs with respect to initial-strings chosen uniformly at ran$d o m$ can be transformed into UOHs with respect to initial-strings chosen arbitrarily. Thus UOHs with respect to initial-strings chosen arbitrarily exist if and only if UOHs with respect to initial-strings chosen uniformly at random exist. The proof is constructive, and may significantly simplify the construction of UOHs with respect to initial-strings chosen arbitrarily, under the assumption of the existence of one-way functions. Then, as an application of the transformation result, we prove that UOHs with respect to initial-strings chosen arbitrarily can be constructed under a weaker assumption, the existence of one-way quasi-injections (whose definition is to be given in Section 5). Next, we investigate relationships among various versions of one-way hash functions. We show that some versions of one-way hash functions are strictly included in others by explicitly constructing hash functions that are one-way in the sense of the former but not in the sense of the latter. A simple method, which appears in [ZMI90], for constructing UOHs from one-way permutations whose (simultaneously) hard bits have been identified is described in Appendix.

\section{Notation and Definitions}

The set of all positive integers is denoted by $N$. Let $\Sigma=\{0,1\}$ be the alphabet we consider. For $n \in \mathbf{N}$, denote by $\Sigma^{n}$ the set of all strings over $\Sigma$ with length $n$, by $\Sigma^{*}$ that of all finite length strings including the empty string, denoted by $\lambda$, over $\Sigma$, and by $\Sigma^{+}$the set $\Sigma^{*}-\{\lambda\}$. The concatenation of two strings $x, y$ is denoted by $x \diamond y$, or simply by $x y$ if no confusion arises. The length of a string $x$ is denoted by $|x|$, and the number of elements in a set $S$ is denoted by $\sharp S$.

Let $\ell$ be a monotone increasing function from $\mathbf{N}$ to $\mathbf{N}$, and $f$ a (total) function from $D$ to $R$, where $D=\cup_{n} D_{n}, D_{n} \subseteq \Sigma^{n}$, and $R=\cup_{n} R_{n}, R_{n} \subseteq \Sigma^{\ell(n)} . D$ is called the domain, and $R$ the range of $f$. For simplicity of presentation, in this paper we always assume that $D_{n}=\Sigma^{n}$ and $R_{n}=\Sigma^{\ell(n)}$. Denote by $f_{n}$ the restriction of $f$ on $\Sigma^{n}$. We are concerned only with the case when the range of $f_{n}$ is $\Sigma^{\ell(n)}$, i.e., $f_{n}$ is a function from $\Sigma^{n}$ to $\Sigma^{\ell(n)}$. $f$ is an injection if each $f_{n}$ is a one-to-one function, and is a permutation if each $f_{n}$ is a one-to-one and onto function. $f$ is (deterministic/probabilistic) 
polynomial time computable if there is a (deterministic/probabilistic) polynomial (in $|x|$ ) time algorithm (Turing machine) computing $f(x)$ for all $x \in D$. The composition of two functions $f$ and $g$ is defined as $f \circ g(x)=f(g(x))$. In particular, the $i$-fold composition of $f$ is denoted by $f^{(i)}$.

A (probability) ensemble $E$ with length $\ell(n)$ is a family of probability distributions $\left\{E_{n} \mid E_{n}: \Sigma^{\ell(n)} \rightarrow[0,1], n \in \mathrm{N}\right\}$. The uniform ensemble $U$ with length $\ell(n)$ is the family of uniform probability distributions $U_{n}$, where each $U_{n}$ is defined as $U_{n}(x)=$ $1 / 2^{\ell(n)}$ for all $x \in \Sigma^{\ell(n)}$. By $x \in \in_{E} \Sigma^{\ell(n)}$ we mean that $x$ is randomly chosen from $\Sigma^{\ell(n)}$ according to $E_{n}$, and in particular, by $x \in_{R} S$ we mean that $x$ is chosen from the set $S$ uniformly at random. $E$ is samplable if there is a (probabilistic) algorithm $M$ that on input $n$ outputs an $x \in \in_{E} \Sigma^{\ell(n)}$, and polynomially samplable if furthermore, the running time of $M$ is polynomially bounded.

Now we introduce the notion for one-way functions, a topic that has received extensive research (see for examples [Yao82] [Wa88] [ILL89]).

Definition 1 Let $f: D \rightarrow R$, where $D=\cup_{n} \Sigma^{n}$ and $R=\bigcup_{n} \Sigma^{\ell(n)}$, be a polynomial time computable function, and let $E$ be an ensemble with length $n$. (1) $f$ is one-way with respect to $E$ if for each probabilistic polynomial time algorithm $M$, for each polynomial $Q$ and for all sufficiently large $n, \operatorname{Pr}\left\{f_{n}(x)=f_{n}\left(M\left(f_{n}(x)\right)\right)\right\}<1 / Q(n)$, when $x \in_{E} \Sigma^{n}$. (2) $f$ is one-way if it is one-way with respect to the uniform ensemble $U$ with length $n$.

There are two basic computation models: Turing machines and combinational circuits (see for examples [Pip79] [KL82] [BDG88]). The above definition for one-way functions is with respect to the Turing machine model. A stronger version of oneway functions that is with respect to the circuit model can be obtained by changing algorithms $M$ in the above definition to families $M=\left\{M_{n} \mid n \in \mathrm{N}\right\}$ of polynomial size circuits.

\section{Universal One-Way Hash Functions}

The central concept treated in this paper is one-way hash functions. Two kinds of one-way hash functions have been considered in the literature: universal one-way hash functions and collision-intractable hash functions (or shortly UOHs and CIHs, respectively). In [Mer89] the former is called weakly and the latter strongly, one-way hash functions respectively. Naor and Yung gave a formal definition for UOH [NY89], and Damgård gave for $\mathrm{CIH}$ [Dam89]. In this section, a formal definition for $\mathrm{UOH}$ that is more general than that of [NY89] is given. We feel our formulation more reasonable. This will be explained after the formulation is introduced. CIH will be treated in later sections. 
Let $\ell$ be a polynomial with $\ell(n)>n, H$ be a family of functions defined by $H=\bigcup_{n} H_{n}$ where $H_{n}$ is a (possibly multi-) set of functions from $\Sigma^{\ell(n)}$ to $\Sigma^{n}$. Call $H$ a hash function compressing $\ell(n)$-bit input into $n$-bit output strings. For two strings $x, y \in \Sigma^{\ell(n)}$ with $x \neq y$, we say that $x$ and $y$ collide with each other under $h \in H_{n}$, or $(x, y)$ is a collision pair for $h$, if $h(x)=h(y)$.

$H$ is polynomial time computable if there is a polynomial (in $n$ ) time algorithm computing all $h \in H$, and accessible if there is a probabilistic polynomial time algorithm that on input $n \in \mathbf{N}$ outputs uniformly at random a description of $h \in H_{n}$. It is assumed that all hash functions considered in this paper are both polynomial time computable and accessible.

Let $H$ be a hash function compressing $\ell(n)$-bit input into $n$-bit output strings, and $E$ an ensemble with length $\ell(n)$. The definition for UOH is best described as a three-party game. The three parties are $S$ (an initial-string supplier), $G$ (a hash function instance generator) and $F$ (a collision-string finder). $S$ is an oracle whose power is un-limited, and both $G$ and $F$ are probabilistic polynomial time algorithms. The first move is taken by $S$, who outputs an initial-string $x \in_{E} \Sigma^{\ell(n)}$ and sends it to both $G$ and $F$. The second move is taken by $G$, who chooses, independently of $x$, an $h \in_{R} H_{n}$ and sends it to $F$. The third and also final (null) move is taken by $F$, who on input $x \in \Sigma^{\ell(n)}$ and $h \in H_{n}$ outputs either "?" (I don't know) or a string $y \in \Sigma^{\ell(n)}$ such that $x \neq y$ and $h(x)=h(y)$. F wins a game iff his/her output is not equal to "?". Informally, $H$ is a universal one-way hash function with respect to $E$ if for any collision-string finder $F$, the probability that $F$ wins a game is negligible. More precisely:

Definition 2 Let $H$ be a hash function compressing $\ell(n)$-bit input into $n$-bit output strings, $P$ a collection of ensembles with length $\ell(n)$, and $F$ a collision-string finder. $H$ is a universal one-way hash function with respect to $P$, denoted by UOH/P, if for each $E \in P$, for each $F$, for each polynomial $Q$, and for all sufficiently large $n$, $\operatorname{Pr}\{F(x, h) \neq ?\}<1 / Q(n)$, where $x$ and $h$ are independently chosen from $\Sigma^{\ell(n)}$ and $H_{n}$ according to $E_{n}$ and to the uniform distribution over $H_{n}$ respectively, and the probability $\operatorname{Pr}\{F(x, h) \neq ?\}$ is computed over $\Sigma^{\ell(n)}, H_{n}$ and the sample space of all finite strings of coin fips that $F$ could have tossed.

If $P$ consists of a single ensemble $E$ (i.e., $P=\{E\}$ ), $\mathrm{UOH} / E$ is synonymous with $\mathrm{UOH} / P$. Of particular interest are the following versions of $\mathrm{UOH}$ : (1) $\mathrm{UOH} / E N[\ell]$,

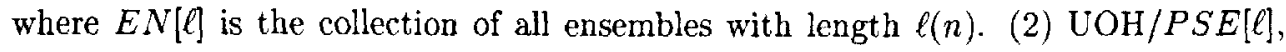
where $P S E[\ell]$ is the collection of all polynomially samplable ensembles with length $\ell(n)$. (3) $\mathrm{UOH} / U$, where $U$ is the uniform ensemble with length $\ell(n)$.

In [NY89], Naor and Yung gave a definition for UOH. They did not separate initial-string ensembles from collision-string finders. Instead, they introduced a probabilistic polynomial time algorithm $A(\cdot, \cdot)$, called a collision adversary that works 
in two stages: At the first stage, the algorithm $A$, on input $(\lambda, \lambda)$ where $\lambda$ denotes the empty string, outputs an initial value (corresponding to our initial-string) $x=A(\lambda, \lambda) \in \Sigma^{\ell(n)}$. At the second stage, it, when given an $h \in H_{n}$, attempts to find a string $y=A(x, h) \in \Sigma^{\ell(n)}$ such that $x \neq y$ and $h(x)=h(y)$.

Thus Naor and Yung defined, in our terms, universal one-way hash function with

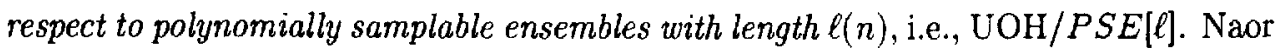

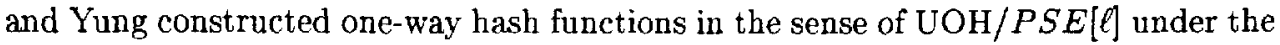
assumption of the existence of one-way injections [NY89]. Note that they actually obtained a construction for one-way hash functions in the sense of UOH/EN[€]. In [ZMI90] we construct, in a different approach, one-way hash functions in the sense of $\mathrm{UOH} / E N[\ell]$ under the assumption of the existence of one-way permutations. See Appendix for the description of the construction.

Separating initial-string ensembles from collision-string finders is conceptually much clearer, and enables us to reduce the problem of constructing one-way hash functions in the sense of $\mathrm{UOH} / E N[\ell]$ (the "strongest" $\mathrm{UOHs}$ ) to that of constructing one-way hash functions in the sense of UOH/U (the "weakest" UOHs). This topic is treated in Section 4.

The above definition for $\mathrm{UOH}$ is with respect to the Turing machine model. As a natural counterpart of $U \mathrm{UOH} / P$, where $P$ is a set of ensembles with length $\ell(n)$, we have $\mathrm{UOH}_{C} / P$, whose definition is obtained simply by changing probabilistic polynomial time algorithms $F$ in Definition 2 to families $F=\left\{F_{n} \mid n \in \mathbf{N}\right\}$ of polynomial size circuits.

The definition for $\mathrm{UOH}$ can also be generalized in another direction: In addition to $x \in \Sigma^{\ell(n)}$ and $h \in H_{n}$, a collision-string finder $F$ is allowed to receive an extra advice string $a$. As before, the output of $F$ is either "?" or a string $y \in \Sigma^{\ell(n)}$ such that $x \neq y$ and $h(x)=h(y)$.

Definition 3 Let $H$ be a hash function compressing $\ell(n)$-bit input into $n$-bit output strings. $H$ is a universal one-way hash function with respect to polynomial length advice, denoted by UOH $/ E N\left[\right.$ poly], if for each pair $\left(Q_{1}, Q_{2}\right)$ of polynomials with $Q_{1}(n) \geq \ell(n)$, for each ensemble $E$ with length $Q_{1}(n)$, for each collision-string finder $F$, and for all sufficiently large $n, \operatorname{Pr}\{F(x, a, h) \neq ?\}<1 / Q_{2}(n)$, where $x \in \Sigma^{\ell(n)}$, $a \in \Sigma^{Q_{1}(n)-\ell(n)}, x \circ a$ and $h$ are independently chosen from $\Sigma^{Q_{1}(n)}$ and $H_{n}$ according to $E_{n}$ and to the uniform distribution over $H_{n}$ respectively, and the probability $\operatorname{Pr}\{F(x, a, h) \neq$ ? $\}$ is computed over $\Sigma^{Q_{1}(n)}, H_{n}$ and the sample space of all finite strings of coin flips that $F$ could have tossed.

Notice the difference between Turing machines taking advice discussed in [Pip79] [KL82] and collision-string finders in our Definition 3. In the former case, advice strings are uniquely determined for each $n \in \mathbf{N}$. While in the latter case, they are generated probabilistically. In Section 7 , we will discuss relationships among various 
versions of one-way hash functions including $\mathrm{UOH} / U, \mathrm{UOH} / P S E[\ell], \mathrm{UOH} / E N[\ell]$, $\mathrm{UOH}_{C} / E N[\theta]$, and $\mathrm{UOH} / E N[p o l y]$.

\section{Transforming $\mathrm{UOH} / U$ into $\mathrm{UOH} / E N[\ell]$}

Let $P_{1}, P_{2}$ be collections of ensembles with length $\ell(n)$. We say that UOH $/ P_{1}$ is transformable into $\mathrm{UOH} / P_{2}$ iff given a one-way hash function $H$ in the sense of $\mathrm{UOH} / P_{1}$, we can construct from $H$ a one-way hash function $H^{\prime}$ in the sense of $\mathrm{UOH} / P_{2}$. The main result of this section is Theorem 1 to be proved below, which states that $\mathrm{UOH} / U$ is transformable into $\mathrm{UOH} / E N[\ell]$. Thus constructing one-way hash functions in the sense of $\mathrm{UOH} / E N[\ell]$ under certain assumptions can be fulfilled in two steps: At the first step, we construct one-way hash functions in the sense of $U O H / U$. This would be easier, since a uniform ensemble would be easier to handle than arbitrary ones. Then at the second step, we apply the proof technique for Theorem 1 to obtain one-way hash functions in the sense of $\mathrm{UOH} / E N[\ell]$.

To prove Theorem 1, we require a function family called an invertible uniformizer. Let $T_{n}$ be a set of permutations over $\Sigma^{\ell(n)}$, and let $T=\cup_{n} T_{n} . T$ is a uniformizer with length $\ell(n)$ if it has the following properties 1,2 and 3. Furthermore, $F$ is invertible if it also has the following property 4 .

1. For each $n$, for each pair of strings $x, y \in \Sigma^{\ell(n)}$, there are exactly $\sharp T_{n} / 2^{\ell(n)}$ permutations in $T_{n}$ that map $x$ to $y$.

2. There is a probabilistic polynomial time algorithm that on input $n$ outputs a $t \in_{R} T_{n}$.

3. There is a polynomial time algorithm that computes all $t \in T$.

4. There is a polynomial time algorithm that computes $t^{-1}$ for all $t \in T$.

The first property implies that for any $n \in \mathrm{N}$ and any $x \in \Sigma^{\ell(n)}$, when $t$ is chosen randomly and uniformly from $T_{n}$, the probability that $t(x)$ coincides with a particular $y \in \Sigma^{\ell(n)}$ is $\left(\sharp T_{n} / 2^{\ell(n)}\right) / \sharp T_{n}=1 / 2^{\ell(n)}$, i.e., $t(x)$ is distributed randomly and uniformly over $\Sigma^{\ell(n)}$.

Now we give a concrete invertible uniformizer with length $\ell(n)$. Note that there is a natural one-to-one correspondence between strings of $\Sigma^{\ell(n)}$ and elements of $G F\left(2^{\ell(n)}\right)$. So we will not distinguish $G F\left(2^{\ell(n)}\right)$ from $\Sigma^{\ell(n)}$. Let $a$ and $b$ be elements of $G F\left(2^{\ell(n)}\right)$ with $a \neq 0$. Then the affine transformation $t$ defined by $t(x)=a \cdot x+b$ is a permutation over $G F\left(2^{\ell(n)}\right)$, where $\cdot$ and + are multiplication and addition over $G F\left(2^{\ell(n)}\right)$ respectively. Denote by $T_{n}$ the set of all the affine transformations on $G F\left(2^{\ell(n)}\right)$ defined as above. Clearly, $\sharp T_{n}=2^{\ell(n)}\left(2^{\ell(n)}-1\right)$, and for any elements $x, y \in G F\left(2^{\ell(n)}\right)$, there are exactly $\left(2^{\ell(n)}-1\right)=\sharp T_{n} / 2^{\ell(n)}$ affine transformations in $T_{n}$ that map $x$ to $y$. In addition, generating $t \in_{R} T_{n}$ is easy, and for all $t \in T$, computing $t$ and $t^{-1}$ are 
simple tasks. Thus $T=U_{n} T_{n}$ is an invertible uniformizer with length $\ell(n)$. In section $5, T$ will once again play a crucial role in constructing one-way hash functions in the sense of $\mathrm{UOH} / E N[\ell]$ from one-way quasi-injections. Now we are ready to prove the following:

Theorem 1 UOH $U$ is transformable into $U O H / E N[\ell] .{ }^{1}$

Proof : Assume that $H$ is a one-way hash function in the sense of $\mathrm{UOH} / U$, where $U$ is the uniform ensemble with length $\ell(n)$. We show how to construct from $H$ a hash function $H^{\prime}$ that is one-way in the sense of $\mathrm{UOH} / E N[\ell]$.

Let $T=\cup_{n} T_{n}$ be an invertible uniformizer with length $\ell(n)$. Given $H$ and $T=$ $\bigcup_{n} T_{n}$, we construct $H^{\prime}$ as follows: $H^{\prime}=\bigcup_{n} H_{n}^{\prime}$, where $H_{n}^{\prime}=\left\{h^{\prime} \mid h^{\prime}=h \circ t, h \epsilon\right.$ $\left.H_{n}, t \in T_{n}\right\}$. We claim that $H^{\prime}$ is one-way in the sense of $\mathrm{UOH} / E N[\ell]$.

Assume for contradiction that $H^{\prime}$ is not one-way in the sense of $\mathrm{UOH} / E N[\ell]$. Then there are a polynomial $Q$, an infinite subset $\mathrm{N}^{\prime} \subseteq \mathrm{N}$, an ensemble $E^{\prime}$ with length $\ell(n)$ and a probabilistic polynomial time algorithm $F^{\prime}$ such that for all $n \in \mathrm{N}^{\prime}$, the algorithm $F^{\prime}$, on input $x^{\prime} \in_{E^{\prime}} \Sigma^{\ell(n)}$ and $h^{\prime} \epsilon_{R} H_{n}^{\prime}$, finds with probability $1 / Q(n)$ a string $y^{\prime} \in \Sigma^{\ell(n)}$ with $x^{\prime} \neq y^{\prime}$ and $h^{\prime}\left(x^{\prime}\right)=h^{\prime}\left(y^{\prime}\right)$. Now we show how to derive from $F^{\prime}$ a collision-string finder $F$ that for all $n \in \mathrm{N}^{\prime}$, on input $x \in_{R} \Sigma^{\ell(n)}$ and $h \in_{R} H_{n}$ where $x$ is produced in a particular way to be described below, outputs with the same probability $1 / Q(n)$ a string $y \in \Sigma^{\ell(n)}$ with $x \neq y$ and $h(x)=h(y)$.

Let $M$ be a probabilistic Turing machine with an oracle $O$ that on input $n$ outputs an $x^{\prime} \in_{E^{\prime}} \Sigma^{\ell(n)} . M$ produces $x \epsilon_{R} \Sigma^{\ell(n)}$ in the following particular way:

1. Query the oracle $O$ with $n$. Denote by $x^{\prime}$ the string answered by $O$. (Note that the oracle $O$ is indispensable, as $E^{\prime}$ may be not samplable.)

2. Generate an $s \in_{R} T_{n}$ using its random tape.

3. Output $x=s\left(x^{\prime}\right)$.

From the first property of the uniformizer $T=\cup_{n} T_{n}$, we know that the ensemble $E_{M}$ defined by the output of $M$ is the uniform ensemble with length $\ell(n)$.

Let $F$ be a probabilistic Turing machine. $F$ uses the same random tape as $M$ 's and its read-only head for the random tape is in the same position as $M$ 's at the outset. On input $x \in_{E_{M}} \Sigma^{\ell(n)}$ and $h \in_{R} H_{n}$, (important note: since $E_{M}$ is the uniform ensemble with length $\ell(n), x \in_{E_{M}} \Sigma^{\ell(n)}$ is equivalent to $\left.x \in_{R} \Sigma^{\ell(n)}\right), F$ works as follows:

1. Generate a $t \in_{R} T_{n}$ using the random tape and in the same way as $M$ does. Since $M$ shares the random tape with $F$, we have $t=s$.

2. Calculate $z=t^{-1}(x)$. Since $t=s$, we have $z=x^{\prime} \in_{E^{\prime}} \Sigma^{\ell(n)}$.

\footnotetext{
${ }^{1}$ De Santis and Yung obtained, independently, this theorem too [DY90].
} 
3. Call $F^{\prime}$ with input $\left(z, h^{\prime}\right)$, where $h^{\prime}=h \circ t$. Note that $h^{\prime} \in_{R} H_{n}^{\prime}$, since $h \in_{R} H_{n}$ and $t \in_{R} T_{n}$.

4. Let $y^{\prime}=F^{\prime}\left(z, h^{\prime}\right)$. Output $y=y^{\prime}$ whenever $y^{\prime}=$ ?, and $y=t\left(y^{\prime}\right)$ otherwise.

Since $F^{\prime}$ is polynomial time bounded, $F$ is also polynomial time bounded. Furthermore, since $t$ is a permutation over $\Sigma^{\ell(n)}$, we have $y \neq$ ? (i.e. $x \neq y$ and $h(x)=h(y)$ ) iff $y^{\prime} \neq$ ? (i.e. $x^{\prime} \neq y^{\prime}$ and $h^{\prime}\left(x^{\prime}\right)=h^{\prime}\left(y^{\prime}\right)$ ). Thus for all $n \in \mathbf{N}^{\prime}, F$ outputs, with the same probability $1 / Q(n)$, a string $y$ such that $x \neq y$ and $h(x)=h(y)$, which implies that $H$ is not a one-way hash function in the sense of $\mathrm{UOH} / U$, a contradiction.

From the above discussions we know that $H^{\prime}$ is indeed a one-way hash function in the sense of $\mathrm{UOH} / E N[\ell]$. This completes the proof.

A significant corollary of Theorem 1 is:

Corollary 1 One-way hash functions in the sense of $U O H / E N[\ell]$ exist iff those in the sense of $U O H / U$ exist.

\section{UOHs Based on a Weakened Assumption}

As an application of Theorem 1, in this section we construct one-way hash functions in the sense of $\mathrm{UOH} / E N[\ell]$ under a weaker assumption - the existence of one-way quasi-injections. Main ingredients of our construction include (1) one-way quasiinjections, (2) universal hash functions with the collision accessibility property, (3) pair-wise independent uniformizers and, (4) invertible uniformizers. Our construction is partially inspired by [NY89].

\subsection{Preliminaries}

Assume that $f$ is a one-way function from $\bigcup_{n} \Sigma^{n}$ to $\bigcup_{n} \Sigma^{\ell(n)}$. A string $x \in \Sigma^{n}$ is said to have a brother if there is a string $y \in \Sigma^{n}$ such that $f_{n}(x)=f_{n}(y)$.

Definition $4 A$ one-way function $f$ is a one-way quasi-injection iff for any polynomial $Q$ and for all sufficiently large $n \in \mathrm{N}, \sharp B_{n} / 2^{n}<1 / Q(n)$ where $B_{n}$ is the collection of all strings in $\Sigma^{n}$ that have brothers.

Let $\ell$ be a polynomial with $\ell(n)>n, S=\cup_{n} S_{n}$ be a hash function compressing $\ell(n)$-bit input into $n$-bit output strings. $S$ is a strongly universal 2 hash function [CW79] [WC81] if for each $n$, for each pairs $\left(x_{1}, x_{2}\right)$ and $\left(y_{1}, y_{2}\right)$ with $x_{1} \neq x_{2}, x_{1}, x_{2} \in$ $\Sigma^{\ell(n)}$ and $y_{1}, y_{2} \in \Sigma^{n}$, there are $\sharp S_{n} /\left(\sharp \Sigma^{n}\right)^{2}$ functions in $S_{n}$ that map $x_{1}$ to $y_{1}$ and $x_{2}$ to $y_{2} . S$ is said to have the collision accessibility property [NY89] if given a pair $(x, y)$ of strings in $\Sigma^{\ell(n)}$ with $x \neq y$ and a requirement that $s(x)=s(y)$, it is possible to generate in polynomial time a function $s \in S_{n}$ such that $s(x)=s(y)$ with equal 
probability over all functions in $S_{n}$ which obey the requirement. Note that strongly universal $_{2}$ hash functions with collision accessibility property are available without any assumption [NY89].

Let $V_{n}$ be a set of permutations over $\Sigma^{\ell(n)}$, and $V=U_{n} V_{n} . V$ is a pair-wise independent uniformizer with length $\ell(n)$ if it has the following three properties.

1. For each $n$, for any pairs of strings $\left(x_{1}, x_{2}\right)$ and $\left(y_{1}, y_{2}\right)$, there are exactly $\sharp V_{n} /\left[2^{\ell(n)}\left(2^{\ell(n)}-1\right)\right]$ permutations in $V_{n}$ that map $x_{1}$ to $y_{1}$ and $x_{2}$ to $y_{2}$, where $x_{1}, x_{2}, y_{1}, y_{2} \in \Sigma^{\ell(n)}, x_{1} \neq x_{2}, y_{1} \neq y_{2}$, and $2^{\ell(n)}\left(2^{\ell(n)}-1\right)$ is the total number of ordered pairs $(x, y)$ with $x \neq y$ and $x, y \in \Sigma^{\ell(n)}$.

2. There is a probabilistic polynomial time algorithm that on input $n$ outputs a $v \in_{R} V_{n}$.

3. There is a polynomial time algorithm that computes all $v \in V$.

Similar to uniformizers defined in Section 4 , the first property implies that for any $n \in \mathbf{N}$ and any $\left(x_{1}, x_{2}\right)$ with $x_{1} \neq x_{2}$ and $x_{1}, x_{2} \in \Sigma^{\ell(n)}$, when $v$ is chosen randomly and uniformly from $V_{n},\left(v\left(x_{1}\right), v\left(x_{2}\right)\right)$ is distributed randomly and uniformly over all ordered pairs $\left(y_{1}, y_{2}\right)$ with $y_{1} \neq y_{2}$ and $y_{1}, y_{2} \in \Sigma^{\ell(n)}$.

Recall the invertible uniformizer $T=\bigcup_{n} T_{n}$ constructed in Section 4. For any $x_{1}, x_{2}, y_{1}, y_{2} \in \Sigma^{\ell(n)}$ with $x_{1} \neq x_{2}$ and $y_{1} \neq y_{2}$, there is exactly one permutation in $T_{n}$ that maps $x_{1}$ to $y_{1}$ and $x_{2}$ to $y_{2}$. Note that $1=2^{\ell(n)}\left(2^{\ell(n)}-1\right) / 2^{\ell(n)}\left(2^{\ell(n)}-1\right)=$ $\sharp T_{n} /\left[2^{\ell(n)}\left(2^{\ell(n)}-1\right)\right]$, which implies that $T$ is a pair-wise independent uniformizer.

\subsection{UOHs from One-Way Quasi-Injections}

Assume that we are given a one-way quasi-injection $f$ from $D$ to $R$ where $D=U_{n} \Sigma^{n}$, $R=\bigcup_{n} \Sigma^{m(n)}$ and $m$ is a polynomial with $m(n) \geq n$. Let $V=\cup_{n} V_{n}$ be a pair-wise independent uniformizer with length $m(n)$, and $S=U_{n} S_{n}$ be a strongly universal 2 hash function that compresses $m(n)$-bit input into $(n-1)$-bit output strings and has the collision accessibility property.

Lemma 1 let $H_{n}=\left\{h \mid h=s \circ v \circ f_{n+1}, s \in S_{n+1}, v \in V_{n+1}\right\}$, and $H=\bigcup_{n} H_{n}$. Then $H$ is a one-way hash function in the sense of UOH/U compressing $(n+1)$-bit input into $n$-bit output strings, under the assumption that $f$ is a one-way quasi-injection.

Proof: Assume for contradiction that $H$ is not one-way in the sense of $\mathrm{UOH} / U$. Then there are a polynomial $Q_{1}$, an infinite subset $\mathbf{N}^{\prime} \subseteq \mathbf{N}$ and a collision-string finder $F$ such that for all $n \in \mathrm{N}^{\prime}$, the finder $F$, on input $x \in_{R} \Sigma^{n+1}$ and $h \in_{R} H_{n}$, outputs with probability at least $1 / Q_{1}(n)$ a string $y \in \Sigma^{n+1}$ with $x \neq y$ and $h(x)=h(y)$. We show that $F$ can be used to construct an algorithm $M$ that for all sufficiently large $n \in \mathbf{N}^{\prime}$, inverts $f_{n+1}$ with probability greater than $1 / 2 Q_{1}(n)$. 
Assume that $w \in_{R} \Sigma^{n+1}$ and $z=f_{n+1}(w)$. On input $z$, the algorithm $M$ runs as follows in trying to compute a $y$ such that $z=f_{n+1}(y)$ :

\section{Algorithm $M$ :}

1. Generate an $x \in_{R} \Sigma^{n+1}$. If $z=f_{n+1}(x)$ then output $y=x$ and halt. Otherwise execute the following steps.

2. Generate a $v \in_{R} V_{n+1}$.

3. Let $u_{1}=v \circ f_{n+1}(x)$ and $u_{2}=v(z)$. Choose a random $s \in S_{n+1}$ such that $s\left(u_{1}\right)=s\left(u_{2}\right)$. This is possible according to the collision accessibility property of $S$.

4. Let $h=s \circ v \circ f_{n+1}$. Call $F$ with input $h$ and $x$, and output $y=F(x, h)$.

First we show that $h$ produced by $M$ is a random element in $H_{n}$. At Step 2, a $v \in_{R} V_{n+1}$ is generated. Since $f_{n+1}(x) \neq z$, from the first property of $V$ we know that ( $\left.v \circ f_{n+1}(x), v(z)\right)$ is distributed randomly and uniformly over all pairs $\left(x_{1}, x_{2}\right)$ with $x_{1} \neq x_{2}$ and $x_{1}, x_{2} \in \Sigma^{m(n+1)}$. At Step $3, s$ is chosen uniformly at random from all those functions in $S_{n+1}$ that map $u_{1}$ and $u_{2}$ to the same string. Consequently, $h=s \circ v \circ f_{n+1}$ is a random element in $H_{n}$.

The running time of $M$ is clearly polynomial in $n$. Next we estimate the probability that $M$ outputs $y$ such that $z=f_{n+1}(y)$. Denote by $\operatorname{Inv}(z)$ the set $\left\{e \mid z=f_{n+1}(e), e \in\right.$ $\left.\Sigma^{n+1}\right\}$. Then $M$ halts at Step 1 iff $x \in \operatorname{Inv}(z)$.

First we note that

$$
\operatorname{Pr}\left\{z=f_{n+1}(y)\right\} \geq \operatorname{Pr}\left\{x \in \Sigma^{n+1}-\operatorname{Inv}(z), x \text { has no brother, } z=f_{n+1}(y)\right\}
$$

where $\operatorname{Pr}\left\{z=f_{n+1}(y)\right\}$ is computed over $\Sigma^{n+1}, \Sigma^{n+1}, V_{n+1}, S_{n+1}$ and the sample space of all finite strings of coin flips that $F$ could have tossed. Note that the two compound events " $x \in \Sigma^{n+1}-\operatorname{Inv}(z), x$ has no brother, $z=f_{n+1}(y)$ " and " $x \in \Sigma^{n+1}-\operatorname{Inv}(z)$, $x$ has no brother, $y \neq$ ?" are in fact the same. So the probability $\operatorname{Pr}\left\{z=f_{n+1}(y)\right\}$ can be estimated via the probability $\operatorname{Pr}\left\{x \in \Sigma^{n+1}-\operatorname{Inv}(z), x\right.$ has no brother, $y \neq$ ? $\}$. Now we focus on the latter. By assumption, we have $\operatorname{Pr}\{y \neq$ ? $\} \geq 1 / Q_{1}(n)$ for all $n \in \mathbf{N}^{\prime}$, where $\operatorname{Pr}\{y \neq ?\}$ is computed over $\Sigma^{n+1}, V_{n+1}, S_{n+1}$ and the sample space of all finite strings of coin flips that $F$ could have tossed. On the other hand,

$$
\begin{aligned}
\operatorname{Pr}\{y \neq ?\}= & \operatorname{Pr}\{x \in \operatorname{Inv}(z), y \neq ?\}+\operatorname{Pr}\left\{x \in \Sigma^{n+1}-\operatorname{Inv}(z), y \neq ?\right\} \\
= & \operatorname{Pr}\{x \in \operatorname{Inv}(z), y \neq ?\}+ \\
& \operatorname{Pr}\left\{x \in \Sigma^{n+1}-\operatorname{Inv}(z), x \text { has a brother, } y \neq ?\right\}+ \\
& \operatorname{Pr}\left\{x \in \Sigma^{n+1}-\operatorname{Inv}(z), x \text { has no brother, } y \neq ?\right\} .
\end{aligned}
$$


Recall that $f$ is one-way. So for all sufficiently large $n \in \mathbf{N}$, we have

$$
\operatorname{Pr}\{x \in \operatorname{Inv}(z), y \neq ?\} \leq \operatorname{Pr}\{x \in \operatorname{Inv}(z)\}<1 / 4 Q_{1}(n) .
$$

Furthermore, for all sufficiently $n$ we have

$$
\operatorname{Pr}\left\{x \in \Sigma^{n+1}-\operatorname{Inv}(z), x \text { has a brother, } y \neq ?\right\} \leq \operatorname{Pr}\{x \text { has a brother }\}<1 / 4 Q_{1}(n),
$$

since $f$ is a one-way quasi-injection. Thus for all sufficiently large $n \in \mathbf{N}^{\prime}$,

$$
\begin{aligned}
\operatorname{Pr}\left\{z=f_{n+1}(y)\right\} \geq & \operatorname{Pr}\left\{x \in \Sigma^{n+1}-\operatorname{Inv}(z), x \text { has no brother, } z=f_{n+1}(y)\right\} \\
= & \operatorname{Pr}\left\{x \in \Sigma^{n+1}-\operatorname{Inv}(z), x \text { has no brother, } y \neq ?\right\} \\
\geq & 1 / Q_{1}(n)-[\operatorname{Pr}\{x \in \operatorname{Inv}(z), y \neq ?\}+ \\
& \left.\operatorname{Pr}\left\{x \in \Sigma^{n+1}-\operatorname{Inv}(z), x \text { has a brother, } y \neq ?\right\}\right] \\
\geq & 1 / Q_{1}(n)-\left[1 / 4 Q_{1}(n)+1 / 4 Q_{1}(n)\right] \\
\geq & 1 / 2 Q_{1}(n) .
\end{aligned}
$$

This contradicts our assumption that $f$ is a one-way quasi-injection, and hence the theorem follows.

Combining Theorem 1 and Lemma 1, we have the following result: $A$ one-way hash function $H^{\prime}$ in the sense of $U O H / E N\left[\ell^{\prime}\right]$, where $\ell^{\prime}$ is defined by $\ell^{\prime}(n)=n+1$, can be constmucted under the assumption that $f$ is a one-way quasi-injection. By an argument analogous to that of Theorem 3.1 of [Dam89], it can be proved that for any polynomial $\ell$, we can construct from $H^{\prime}$ a one-way hash function $H^{\prime \prime}$ in the sense of $\mathrm{UOH} / E N[\ell]$. Thus:

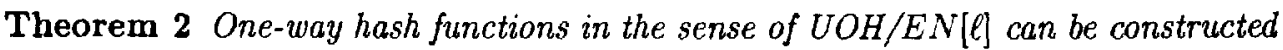
assuming the existence of one-way quasi-injections.

Similarly, we can construct one-way hash functions in the sense of $\mathrm{UOH}_{C} / E N[\ell]$ assuming the existence of one-way quasi-injections with respect to the circuit model.

\section{Collision Intractable Hash Functions}

This section gives formal definitions for collision intractable hash functions. Let $H=\bigcup_{n} H_{n}$ be a hash function compressing $\ell(n)$-bit input into $n$-bit output strings. Let $A$, a collision-pair finder, be a probabilistic polynomial time algorithm that on input $h \in H_{n}$ outputs either "?" or a pair of strings $x, y \in \Sigma^{\ell(n)}$ with $x \neq y$ and $h(x)=h(y)$.

Definition $5 \mathrm{H}$ is called a collision-intractable hash function (CIH) if for each $A$, for each polynomial $Q$, and for all sufficiently large $n, \operatorname{Pr}\{A(h) \neq ?\}<1 / Q(n)$, where $h \in_{R} H_{n}$, and the probability $\operatorname{Pr}\{A(h) \neq$ ? $\}$ is computed over $H_{n}$ and the sample space of all finite strings of coin fips that $A$ could have tossed. 
In [Dam89] (see also [Dam87]) CIH is called collision free function family. Damgård obtained CIHs under the assumption of the existence of claw-free pairs of permutations. In [ZMI90], we show that CIHs can be constructed from distinction-intractable permutations. We also propose practical CIHs, the fastest of which compress nearly $2 n$-bit long input into $n$-bit long output strings by applying only twice a one-way function.

$\mathrm{CIH}$ defined above are with respect to the Turing machine model. So as in the case for $\mathrm{UOH}$, we have $\mathrm{CIH}_{C}$ with respect to the circuit model. The definition for $\mathrm{CIH}_{C}$ is similar to Definition 5, except that probabilistic polynomial time algorithms $A$ are replaced by families $A=\left\{A_{n} \mid n \in \mathbf{N}\right\}$ of polynomial size circuits.

In addition, analogous to Definition 3 , we have the following generalization for CIH. Let $H=\bigcup_{n} H_{n}$ be a hash function compressing $\ell(n)$-bit input into $n$-bit output strings, $Q_{1}$ a polynomial, and $a \in \Sigma^{Q_{1}(n)}$. $a$ is called an advice string of length $Q_{1}(n)$. Let $A$, a collision-pair finder, be a probabilistic polynomial time algorithm that on input $a \in \Sigma^{Q_{1}(n)}$ and $h \in H_{n}$ outputs either "?" or a pair of strings $x, y \in \Sigma^{\ell(n)}$ with $x \neq y$ and $h(x)=h(y)$.

Definition $6 H$ is called a collision intractable hash function with respect to polynomial length advice, denoted by $C I H / E N\left[\right.$ poly], if for each pair $\left(Q_{1}, Q_{2}\right)$ of polynomials, for each ensemble $E$ with length $Q_{1}(n)$, for each $A$, and for all sufficiently large $n, \operatorname{Pr}\{A(a, h) \neq ?\}<1 / Q_{2}(n)$, where $a$ and $h$ are independently chosen from $\Sigma^{Q_{1}(n)}$ and $H_{n}$ according to $E_{n}$ and to the uniform distribution over $H_{n}$ respectively, and the probability $\operatorname{Pr}\{A(a, h) \neq ?\}$ is computed over $\Sigma^{Q_{1}(n)}, H_{n}$ and the sample space of all finite strings of coin fips that $A$ could have tossed.

\section{A Hierarchy of One-Way Hash Functions}

In this section, we discuss relationships among various versions of one-way hash functions: $\mathrm{UOH} / U, \mathrm{UOH} / P S E[\ell], \mathrm{UOH} / E N[\ell], \mathrm{UOH}_{C} / E N[\ell], \mathrm{UOH} / E N[$ poly], CIH, $\mathrm{CIH}_{C}$ and $\mathrm{CIH} / E N[p o l y]$.

First we define a relation between two versions, $V e r_{1}$ and $V e r_{2}$, of one-way hash functions. We say that

1. $V e r_{1}$ is included in $V e r_{2}$, denoted by $V e r_{1} \subseteq V e r_{2}$, if all one-way hash functions in the sense of $V e r_{1}$ are also one-way hash functions in the sense of $V e r_{2}$.

2. $V e r_{1}$ is strictly included in $V e r_{2}$, denoted by $V e r_{1} \subset V e r_{2}$, if $V e r_{1} \subseteq V e r_{2}$ and there is a one-way hash function in the sense of $V e r_{2}$ but not in the sense of $V e r_{1}$.

3. $V e r_{1}$ and $V e r_{2}$ are equivalent, denoted by $V e r_{1}=V e r_{2}$, if $V e r_{1} \subseteq V e r_{2}$ and $V e r_{2} \subseteq V e r_{1}$. 
Lemma 2 The following statements hold:

(1) $C I H_{C}=C I H / E N[p o l y]$.

(2) $U O H_{C} / E N[\ell]=U O H / E N[$ poly $]$.

(3) $U O H / E N[$ poly $] \subseteq U O H / E N[\ell] \subseteq U O H / P S E[\ell] \subseteq U O H / U$.

(4) $C I H / E N[$ poly $] \subseteq C I H$.

(5) $C I H \subseteq U O H / P S E[\ell]$.

(6) $C I H / E N[$ poly $] \subseteq U O H / E N[$ poly $]$.

Proof: Proofs for (1) and (2) are analogous to that for "polynomial size circuits vs. P/poly" [Pip79]. (3),(4), (5) and (6) are obvious. Here we give a detailed description for the proof of (1). Proof for (2) is similar, and is omitted.

The " $\subseteq$ " part: Assume that $H$ is a one-way hash function in the sense of $\mathrm{CIH}_{C}$. If $H$ is not one-way in the sense of $\mathrm{CIH} / E N[p o l y]$, then there are polynomials $Q_{1}$ and $Q_{2}$, an infinite subset $\mathbf{N}^{\prime} \subseteq \mathrm{N}$, an ensemble $E$ with length $Q_{2}(n)$, and a collision-pair finder $F$, such that for all $n \in \mathbf{N}^{\prime}$, the finder $F$, on input $z \in_{E} \Sigma^{Q_{2}(n)}$ and $h \in_{R} H_{n}$, outputs a collision-pair with probability $1 / Q_{1}(n)$. Note that for each $n \in \mathbf{N}$ and $h \in_{R} H_{n}$, the probability that $F$ successfully outputs a collision-pair is computed over $\Sigma^{Q_{2}(n)}$ and the sample space of all finite strings of coin flips that $F$ could have tossed. Let $z_{\max }$ be the first string according to the lexicographic order in $\Sigma^{Q_{2}(n)}$ such that for $h \in_{R} H_{n}, F$ outputs a collision-pair with the maximum probability, which is certainly at least $1 / Q_{1}(n) . F$ can be converted into a family $A=\left\{A_{n} \mid n \in \mathbb{N}\right\}$ of probabilistic polynomial size circuits with $z_{\max }$ being "embedded in" $A_{n}$. Thus for each $n \in \mathbf{N}^{\prime}$, $A_{n}$ on input $h \in_{R} H_{n}$ outputs a collision-pair with probability at least $1 / Q_{1}(n)$. In other words, $H$ is not one-way in the sense of $\mathrm{CIH}_{C}$, which is a contradiction.

The "?" part: Assume that $H$ is a one-way hash function in the sense of CIH/EN[poly]. If $H$ is not one-way in the sense of $\mathrm{CIH}_{C}$, then there are a polynomial $Q_{1}$, an infinite subset $\mathbf{N}^{\prime} \subseteq \mathbf{N}$, and a collision-pair finder $A=\left\{A_{n} \mid n \in \mathbf{N}\right\}$, such that for all $n \in \mathbf{N}^{\prime}, A_{n}$ outputs a collision-pair with probability $1 / Q_{1}(n)$. Since the size of $A$ is polynomially bounded, there is a polynomial $Q_{2}$ such that the description of $A_{n}$ is not longer than $Q_{2}(n)$ for all $n \in \mathrm{N}$. Without loss of generality, assume that the description of $A_{n}$ is exactly $Q_{2}(n)$ bits long. Let $E$ be the ensemble with length $Q_{2}(n)$ defined by $E_{n}(x)=1$ whenever $x$ is the description of $A_{n}$, and $E_{n}(x)=0$ otherwise. Note that $E$ may be not samplable.

Recall that the (probabilistic) circuit value problem is (probabilistic) polynomial time computable (see [BDG88], p.110). So there is a (probabilistic) polynomial time algorithm $F$ that on input $z \in_{E} \Sigma^{Q_{2}(n)}$ and $h \in_{R} H_{n}$, (Note: By the definition of $E$, we have $z=$ the description of $A_{n}$ ), output a collision-pair with probability $1 / Q(n)$. 
This implies that $H$ is not one-way in the sense of CIH/EN[poly], which contradicts our assumption.

Theorem 3 The following statements hold:

(1) $U O H / P S E[\ell] \subset U O H / U$.

(2) There are one-way hash functions in the sense of UOH/EN[poly] but not in the sense of $\mathrm{CIH}$.

(3) $C I H \subset U O H / P S E[\ell]$.

(4) $C I H / E N[p o l y] \subset U O H / E N[p o l y]$.

Proof : (1) We show that given a one-way hash function $H$ in the sense of $\mathrm{UOH} / U$, we can construct from $H$ a hash function $H^{\prime}$ that is still one-way in the sense of $\mathrm{UOH} / U$ but not in the sense of $\mathrm{UOH} / P S E[\ell]$.

$H^{\prime}$ is constructed as follows: Denote by $0^{\ell(n)}\left(1^{\ell(n)}\right.$, respectively) the all-0 (all-1, respectively) string of length $\ell(n)$. For each $h \in H_{n}$, define a function $h^{\prime}: \Sigma^{\ell(n)} \rightarrow \Sigma^{n}$ by $h^{\prime}(x)=h\left(0^{\ell(n)}\right)$ whenever $x=1^{\ell(n)}$ and $h^{\prime}(x)=h(x)$ otherwise. Thus the only difference between $h$ and $h^{\prime}$ is the images of $1^{\ell(n)}$. Let $H_{n}^{\prime}$ be the collection of all $h^{\prime}$, and let $H^{\prime}=\bigcup_{n} H_{n}^{\prime}$. We claim that $H^{\prime}$ is still one-way in the sense of $\mathrm{UOH} / U$ but not in the sense of UOH/PSE[ [] .

Let $M$ be a polynomial time algorithm that on input $n$ outputs $1^{\ell(n)}$. By definition, the ensemble $E$ defined by the output of $M$ is polynomially samplable. Let $F$ be a collision-string finder that on input $x$ and $h^{\prime}$ outputs the string $0^{\ell(n)}$ whenever $x=1^{\ell(n)}$ and "?" otherwise. Clearly, for all $n, x \in_{E} \Sigma^{(\ell(n)}$ and $h^{\prime} \in H_{n}^{\prime}, F$ always finds a string

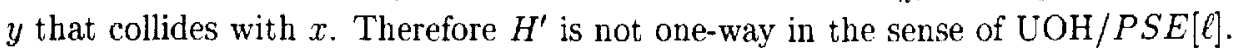

Now we prove that $H^{\prime}$ is one-way in the sense of $\mathrm{UOH} / U$. Assume for contradiction that $H^{\prime}$ is not one-way in the sense of $\mathrm{UOH} / U$. Then there are an infinite subset $\mathrm{N}^{\prime} \subseteq \mathrm{N}$ and a collision-string finder $F$ such that for some polynomial $Q$ and for all $n \in \mathbf{N}^{\prime}, \operatorname{Pr}\left\{F\left(x, h^{\prime}\right) \neq ?\right\} \geq 1 / Q(n)$, when $x \in_{R} \Sigma^{\ell(n)}$ and $h^{\prime} \in_{R} H_{n}^{\prime}$.

Note that

$$
\begin{aligned}
\operatorname{Pr}\left\{F\left(x, h^{\prime}\right) \neq ?\right\} \\
=\operatorname{Pr}\left\{F\left(x, h^{\prime}\right) \neq ? \mid h^{\prime}(x)=h^{\prime}\left(0^{\ell(n)}\right)\right\} \cdot \operatorname{Pr}\left\{h^{\prime}(x)=h^{\prime}\left(0^{\ell(n)}\right)\right\}+ \\
\quad \operatorname{Pr}\left\{F\left(x, h^{\prime}\right) \neq ? \mid h^{\prime}(x) \neq h^{\prime}\left(0^{\ell(n)}\right)\right\} \cdot \operatorname{Pr}\left\{h^{\prime}(x) \neq h^{\prime}\left(0^{\ell(n)}\right)\right\} \\
\geq 1 / Q(n),
\end{aligned}
$$


and that

$$
\begin{aligned}
& \operatorname{Pr}\left\{F\left(x, h^{\prime}\right) \neq ? \mid h^{\prime}(x)=h^{\prime}\left(0^{\ell(n)}\right)\right\} \cdot \operatorname{Pr}\left\{h^{\prime}(x)=h^{\prime}\left(0^{\ell(n)}\right)\right\} \\
& \quad \leq \operatorname{Pr}\left\{h^{\prime}(x)=h^{\prime}\left(0^{\ell(n)}\right)\right\} \\
& \quad \leq \operatorname{Pr}\left\{h(x)=h\left(0^{\ell(n)}\right)\right\}+1 / 2^{\ell(n)} \\
& \quad \leq 2 \operatorname{Pr}\left\{h(x)=h\left(0^{\ell(n)}\right)\right\} .
\end{aligned}
$$

Since $H$ is one-way in the sense of $\mathrm{UOH} / U$, we have $\operatorname{Pr}\left\{h(x)=h\left(0^{\ell(n)}\right)\right\}<1 / 4 Q(n)$ for all sufficiently large $n$. Thus for all sufficicntly large $n \in \mathbf{N}^{\prime}$,

$$
\begin{aligned}
& \operatorname{Pr}\left\{F\left(x, h^{\prime}\right) \neq ? \mid h^{\prime}(x) \neq h^{\prime}\left(0^{\ell(n)}\right)\right\} \\
& \quad \geq \operatorname{Pr}\left\{F\left(x, h^{\prime}\right) \neq ? \mid h^{\prime}(x) \neq h^{\prime}\left(0^{\ell(n)}\right)\right\} \cdot \operatorname{Pr}\left\{h^{\prime}(x) \neq h^{\prime}\left(0^{\ell(n)}\right)\right\} \\
& \quad \geq 1 / Q(n)-\operatorname{Pr}\left\{F\left(x, h^{\prime}\right) \neq ? \mid h^{\prime}(x)=h^{\prime}\left(0^{\ell(n)}\right)\right\} \cdot \operatorname{Pr}\left\{h^{\prime}(x)=h^{\prime}\left(0^{\ell(n)}\right)\right\} \\
& \quad>1 / 2 Q(n) .
\end{aligned}
$$

By definition, when $h^{\prime}(x) \neq h^{\prime}\left(0^{\ell(n)}\right)$, a string $y \in \Sigma^{\ell(n)}$ with $x \neq y$ collides with $x$ under $h^{\prime}$ iff it does under $h$. Consequently, the collision-string finder $F$ can be used to "break" $H$, this implies that $H$ is not one-way in the sense of $\mathrm{UOH} / U$, a contradiction.

(2) The proof is very similar to that for (1). Given $H$, a one-way hash function in the sense of $\mathrm{UOH} / E N\left[\right.$ poly], we construct a hash function $H^{\prime}$ that is still one-way in the sense of $\mathrm{UOH} / E N[$ poly] but not in the sense of $\mathrm{CIH}$.

Without loss of generality, assume that the length of the description of $h \in H_{n}$ is greater than $n / 2$, and for any distinct $h_{1}, h_{2} \in H_{n}$ the first $n / 2$ bits of $h_{1}$ is different from that of $h_{2}$. For each $h \in H_{n}$, we associate with it a particular $\ell(n)$-bit string $x_{h}$ that is obtained by repeatedly concatenating the first $n / 2$ bits of the description of $h$ until the length of the resulting string becomes $\ell(n)$.

For each $h \in H_{n}$, define a function $h^{\prime}: \Sigma^{\ell(n)} \rightarrow \Sigma^{n}$ by $h^{\prime}(x)=h\left(x_{h}\right)$ whenever $x=\bar{x}_{h}$ and $h^{\prime}(x)=h(x)$ otherwise, where $\bar{x}_{h}$ is the complement of $x_{h}$. Thus the only difference between $h$ and $h^{\prime}$ is the images of $\bar{x}_{h}$. Let $H_{n}^{\prime}$ be the collection of all $h^{\prime}$, and let $H^{\prime}=\bigcup_{n} H_{n}^{\prime}$. By analyses similar to (1), one can verify that $H^{\prime}$ is still one-way in the sense of $\mathrm{UOH} / E N[$ poly $]$ but not in the sense of $\mathrm{CIH}$.

(3) follows from (2) and $C I H \subseteq \mathrm{UOH} / P S E[\ell]$. (4) follows from (2) and the facts that $\mathrm{CIH} / E N[$ poly $] \subseteq C I H$ and that $\mathrm{CIH} / E N[$ poly $] \subseteq \mathrm{UOH} / E N[$ poly $]$.

From Lemma 2 and Theorem 3, we have the following hierarchical structure for one-way hash functions (see Figure 1.) 


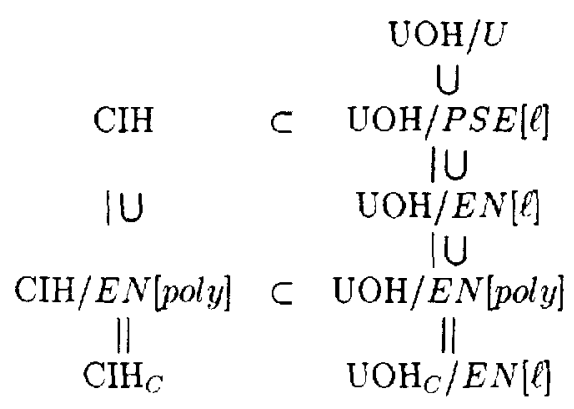

Figure 1. Hierarchical Structure of One-Way Hash Functions

By Theorem 3, there are one-way hash functions in the sense of UOH/EN[poly] but not in the sense of $\mathrm{CIH}$. However, it is not clear whether or not $\mathrm{CIH} \subseteq \mathrm{UOH} / E N[p o l y]$. So it is worth while examining such problems as whether or not CIH is strictly included in $\mathrm{UOH} / E N[$ poly].

\section{Conclusions}

We have proved that UOHs with respect to initial-strings chosen uniformly at random can be transformed into UOHs with respect to initial-strings chosen arbitrarily, and that UOHs with respect to initial-strings chosen arbitrarily can be constructed under a weaker assumption, the existence of one-way quasi-injections. We have also investigated relationships among various versions of one-way hash functions. In particular, we have shown that $\mathrm{UOH} / P S E[\ell], \mathrm{CIH}$ and $\mathrm{CIH} / E N[p o l y]$ are strictly included in $\mathrm{UOH} / U, \mathrm{UOH} / P S E[\ell]$ and $\mathrm{UOH} / E N[$ poly] respectively, and that there are one-way hash functions in the sense of $\mathrm{UOH} / E N[$ poly] but not in the sense of CIH.

Recently, substantial progress on the construction of UOHs has been made by De Santis and Yung [DY90], and especially, by Rompel [Rom90] who finally solved the problem of constructing UOHs under the sole assumption of the existence of one-way functions.

Acknowledgments We would like to thank J. Leo, M. Ogiwara, K. Ohta and K. Sakurai for their fruitful discussions.

\section{References}

[BDG88] J. Balcázar, J. Díaz and J. Gabarró: Structural Complexity I, EATCS Monographs on Theoretical Computer Science, Springer-Verlag, Berlin, 1988. 
[CW79] J. Carter and M. Wegman: "Universal classes of hash functions", Journal of Computer and System Sciences, Vol.18, 1979, pp.143-154.

[Dam87] I. Damgård: "Collision free hash functions and public key signature schemes", Proceedings of EuroCrypt'87, 1987, pp.203-216.

[Dam89] I. Damgård: "A design principle for hash functions", Presented at Crypto' $89,1989$.

[DY90] A. De Santis and M. Yung: "On the design of provably-secure cryptographic hash functions", Presented at EuroCrypt'90, 1990.

[ILL89] R. Impagliazzo, L. Levin and M. Luby: "Pseudo-random generation from one-way functions", Proceedings of the 21-th ACM Symposium on Theory of Computing, 1989, pp.12-24.

[KL82] R. Karp and R. Lipton: "Turing machines that take advice", L'enseigment Mathematique, Vol.28, 1982, pp.191-209.

[Mer89] R. Merkle: "One way hash functions and DES", Presented at Crypto'89, 1989.

[NY89] M. Naor and M. Yung: "Universal one-way hash functions and their cryptographic applications", Proceedings of the 21-th ACM Symposium on Theory of Computing, 1989, pp.33-43.

[Pip79] N. Pippenger: "On simultaneous resource bounds", Proceedings of the 20-th IEEE Symposium on the Foundations of Computer Science, 1979, pp.307311.

[Rom90] J. Rompel: "One-way functions are necessary and sufficient for secure signatures", Proceedings of the 22-nd ACM Symposium on Theory of Computing, 1990, pp.387-394.

[Wa88] O. Watanabe: "On one-way functions", Presented at the International Symposium on Combinatorial Optimization, Tianjin, China, 1988.

[WC81] M. Wegman and J. Carter: "New hash functions and their use in authentication and set equality", Journal of Computer and System Sciences, Vol.22, 1981, pp.265-279.

[Ya082] A. Yao: "Theory and applications of trapdoor functions", Proceedings of the 23-rd IEEE Symposium on the Foundations of Computer Science, 1982, pp.80-91. 
[ZMI90] Y. Zheng, T. Matsumoto and H. Imai: "Duality between two cryptographic primitives", To be presented at 8-th International Conference on Applied Algebra, Algebraic Algorithms and Error Correcting Codes (AAECC-8), Tokyo, August 1990. A preliminary version appears in IEICE Technical Reports on Information Security, TG ISEC89-46, March 16, 1990.

\section{A Appendix - UOHs from One-Way Permuta- tions}

In this appendix we sketch a simple method, which appears in [ZMI90], for constructing UOHs from one-way permutations whose (simutaneously) hard bits have been identified. An interesting feature of our construction is that it does not apply universal hash functions, and hence is extremely compact, in comparison with most of the currently known constructions.

Assume that $f$ is a one-way permutation on $D=\bigcup_{n} \Sigma^{n}$, and that $i$ has been proved to be a hard bit of $f$. For $b \in \Sigma, x \in \Sigma^{n-1}$ and $y \in \Sigma^{n}$, define ins $(x, b)=$ $x_{n-1} x_{i-2} \cdots x_{i} b x_{i-1} \cdots x_{2} x_{1}$, and denote by $\operatorname{drop}(y)$ a function dropping the $i$-th bit of $y$. Then we have the following theorem.

Theorem 4 Let $\ell$ be a polynomial with $\ell(n)>n, \alpha \in \Sigma^{n-1}$ and $x=x_{\ell(n)} \cdots x_{2} x_{1}$ where $x_{i} \in \Sigma$ for each $1 \leq i \leq \ell(n)$. Let $h_{\alpha}$ be the function from $\Sigma^{\ell(n)}$ to $\Sigma^{n}$ defined by:

$$
\begin{aligned}
y_{0} & =\alpha \\
y_{1} & =\operatorname{drop}\left(f_{n}\left(\operatorname{ins}\left(y_{0}, x_{\ell(n)}\right)\right)\right), \\
& \ldots \\
y_{j} & =\operatorname{drop}\left(f_{n}\left(\operatorname{ins}\left(y_{j-1}, x_{\ell(n)-j+1}\right)\right)\right), \\
& \cdots \\
h_{\alpha}(x) & =f_{n}\left(\operatorname{ins}\left(y_{\ell(n)-1}, x_{1}\right)\right) .
\end{aligned}
$$

Let $H_{n}=\left\{h_{\alpha} \mid \alpha \in \Sigma^{n-1}\right\}$ and $H=\cup_{n} H_{n}$. Then under the assumption that $f$

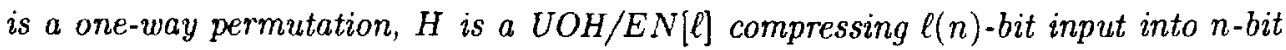
output strings.

The efficiency of the above constructed UOHs can be improved by a factor of $\beta$, for any $\beta=\mathrm{O}(\log n)$, if $\beta$ simultaneously hard bits of $f$ have been identified. 